\title{
Complejidad e inclusividad del comportamiento intrasituacional: Análisis empirico
}

\author{
Victor Hugo González-Becerra; Jairo Ernesto Tamayo Tamayo; Felipe de Jesús Patrón Espinosa; \\ Carlos de Jesús Torres Ceja; Gerardo Alfonso Ortiz Rueda
}

How to cite this article:

González-Becerra, V.H., Tamayo Tamayo, J.E., Patrón Espinosa, F.de J., Torres Ceja, C.de J., \& Ortiz Rueda, G.A. (2020). Complexity and inclusivity of intrasituational behavior: empirical analysis. Acta Colombiana de Psicología, 23(2), 289-308. http://doi.org/10.14718/ACP.2020.23.2.12

Recibido, septiembre 04/2018; Concepto de evaluación, octubre 24/2018; Aceptado, enero 16/2020

\author{
Victor Hugo González-Becerra* \\ Universidad de Guadalajara, Guadalajara, México \\ ORCID: https://orcid.org/0000-0001-7744-6543 \\ Jairo Ernesto Tamayo Tamayo \\ Universidad Veracruzana, Xalapa-Enríquez, México \\ ORCID: https://orcid.org/0000-0001-7738-1786 \\ Felipe de Jesús Patrón Espinosa \\ Universidad Autónoma de Baja California, Mexicali, México \\ ORCID: https://orcid.org/0000-0003-0340-5751 \\ Carlos de Jesús Torres Ceja \\ Universidad de Guadalajara, Guadalajara, México \\ ORCID: https://orcid.org/0000-0002-4279-8490 \\ Gerardo Alfonso Ortiz Rueda \\ Universidad de Guadalajara, Guadalajara, México \\ ORCID: https://orcid.org/0000-0002-8290-2176
}

\begin{abstract}
Resumen
En la taxonomía de Ribes y López (1985) se propone que el comportamiento psicológico es progresivamente complejo e inclusivo; sin embargo, en la literatura sobre el tema se encuentran pocas investigaciones y los datos no son robustos. Teniendo esto en cuenta, y con el propósito de aumentar la evidencia de la complejidad e inclusividad de los tres primeros niveles de complejidad conductual de la taxonomía en tres secuencias de entrenamiento -ascendente (contextual-suplementario-selector), descendente-ascendente (suplementario-contextual-selector) y descendente (selectorsuplementario-contextual) - el presente estudio tuvo como objetivo evaluar el efecto de la historia de interacción — por medio de secuencias de entrenamiento - sobre el proceso de ajuste diferencial (contextual), efectivo (suplementario) y preciso (selector). En general, los resultados muestran que cuando los participantes carecían de historia de interacción ante las contingencias programadas se requirió de un mayor número de sesiones de entrenamiento para mejorar el desempeño en organizaciones funcionales de mayor complejidad; y que cuando la historia de interacción estaba presente en la organización funcional —en tanto interacción previa con las contingencias - se encontró un efecto de facilitación en el ajuste conductual, independientemente de si la transición fue ascendente o descendente. Al final se indaga sobre si el incremento en el número de sesiones se relaciona con la complejidad de cada nivel de organización funcional, y se discute, respecto a las transiciones de entrenamiento funcional, ascendente y descendente, sus efectos en la facilitación en el aprendizaje y su relación con el supuesto de inclusividad funcional.

Palabras clave: interconducta, inclusividad, complejidad, aprendizaje, facilitación, intrasituacional.
\end{abstract}

\footnotetext{
Centro Universitario de los Valles, Carretera Guadalajara-Ameca, Km 45.5, C. P.: 46600, Ameca, Jalisco, México. Tel.: +52 (375) 7580 500, ext.9. victor.becerra@valles.udg.mx
} 


\section{Complexity and inclusivity of intrasituational behavior: empirical analysis}

\begin{abstract}
Ribes and López's (1985) taxonomy proposed that psychological behavior is progressively complex and inclusive. In that respect, there is little research and data are not robust. A study was conducted with the purpose to increase data related to the complexity and inclusivity of the three less complex behaviors of the taxonomy, with three training sequences, namely: 1) ascending (contextual-supplementary-selector), 2) descending-ascending (supplementary -contextual-selector) and 3) descending (selector-supplementary-contextual). The objective was to evaluate the effect of the interaction history (related to training sequences) on differential (contextual), effective (supplementary) and precise (selector) behavior adjustment process. Results showed that a greater number of training sessions were required to improve the performance in functional organizations with greater complexity when participants lacked an interaction history related to programmed contingencies. But, when the interaction history participated in the functional organization, as a previous interaction with the contingencies, a facilitating effect was found in the behavioral adjustment, regardless of whether the transition was ascending or descending. It is discussed whether the increase in the number of sessions is related to the complexity of each level of functional organization. Regarding the functional training transitions, ascending and descending, its effects on facilitation in learning are discussed in relation to the assumption of functional inclusivity.

Keywords: interbehavior, inclusivity, complexity, learning, facilitation, intrasituational.
\end{abstract}

\section{Introducción}

En el desarrollo ontogenético de un organismo, la historia de interacción con los objetos y eventos del ambiente es el factor diferenciador entre el comportamiento psicológico y el biológico. De entre los distintos sistemas teóricos para el análisis del comportamiento psicológico, el interconductismo propone su estudio con base en el sistema de contingencias - es decir, relaciones de dependenciaque se estructuran en la interacción, denominada campo o función psicológica (Kantor, 1924, 1926, 1959). Bajo esta lógica, Ribes y López (1985) plantearon el estudio del comportamiento psicológico a través de una taxonomía de cinco niveles de organización funcional progresivamente complejos e inclusivos. De menor a mayor grado, dichos niveles se denominan: (a) contextual, (b) suplementario, (c) selector, (d) sustitutivo referencial y (e) sustitutivo no referencial. Esta forma de conceptualizar el comportamiento psicológico permite estudiar la evolución cualitativa y cuantitativa de cada tipo de interacción, reconociendo sus elementos diferenciadores y vinculantes (Ribes-Iñesta, 2004, 2007).

En general, la teoría interconductual supone que lo psicológico no está en el organismo ni en su acción, sino en el sistema interactivo en el que este participa. En este sentido, de acuerdo con Ribes y López (1985), la organización funcional del comportamiento psicológico puede configurarse de maneras cualitativamente diferentes con respecto al sistema de contingencias involucrado en la interacción organismo-ambiente; $y$, de todos los elementos que participan en la interacción, podrían considerarse tres de ellos como los más relevantes en la diferenciación de la estructura cualitativa de cada nivel de organización funcional, a saber: (a) el mediador, elemento crítico en la estructuración del sistema de contingencias implicado en la función estímulo-respuesta; (b) el desligamiento funcional, forma en la cual la respuesta se diversifica, transforma y amplía funcionalmente, propiciando un tipo particular de interacción en virtud de la capacidad reactiva del organismo - dada por su ontogenia y filogenia - y las características del ambiente - dadas independiente o interdependientemente de la actividad del organismo-; y (c) el tipo de ajuste conductual, que supone la forma en que la respuesta se ajusta funcionalmente al sistema de contingencias en el cual el organismo o individuo interactúa (Ribes-Iñesta, 2004, 2007; Ribes \& López, 1985).

Los tres primeros tipos de organización funcional planteados por Ribes y López (1985) — contextual, suplementario y selector - se caracterizan porque, en las interacciones en las que el organismo participa, su reactividad se encuentra ligada a las contingencias de la situación — siendo un sistema interactivo-, pero sin trascenderlas, motivo por el cual se les denomina funciones intrasituacionales. Específicamente, la situación puede incluir eventos físicoquímicos, ecológicos o convencionales, cuyas propiedades de estímulo pueden ser constantes o cambiantes (relativas), según se requiera en la interacción. Así, las relaciones de condicionalidad entre acontecimientos potenciales o efectivos vinculados con el comportamiento del organismo o individuo establecen la situación de interacción, siendo secundario el lugar particular donde el comportamiento ocurre (Torres et al., 2012).

En el caso de los dos últimos tipos de organización funcional — sustitutivo referencial y sustitutivo no referencial—, 
la conducta del organismo se desliga de una situación particular para transitar a o construir, respectivamente, un sistema contingencial o situación de naturaleza distinta; además, respecto a estas dos últimas funciones, cabe destacar que su reactividad característica es posible solo en organismos humanos que se desarrollan en ambientes lingüísticos-sociales, dado que no es suficiente que varios organismos interactúen entre sí para que el ambiente sea social, sino que este es social solo si la respuesta a los objetos o individuos es convencional - en el sentido en que quienes participan de la interacción acuerdan implícita o explícitamente la repuesta conjunta a estos-(Ribes-Iñesta, 2001; Ribes-Iñesta et al., 2008).

Ahora bien, tras poco más de tres décadas del origen de esta taxonomía, el escrutinio empírico de datos relacionados con la complejidad e inclusividad de las funciones psicológicas es escaso. Incluso, hay un gran número de casos de funciones psicológicas planteadas por Ribes y López (1985) y Ribes-Iñesta (2007) que no cuentan con datos empíricos, como sucede con el caso de la referencia del referidor y el caso de las contingencias de eventos en tiempos distintos que ocurren en una misma ubicación espacial.

Ahora, si bien los problemas de correspondencia entre las teorías y los fenómenos que describen o explican se "resuelven" a través de análisis conceptuales y lógicos, la investigación empírica permite refutar o aceptar las hipótesis particulares que derivan de los planteamientos generales de una teoría, de modo que ambas tareas - la teórica y la empírica- son igual de relevantes en el quehacer científico. Es en este sentido que la presente investigación tuvo como propósito el análisis de datos empíricos que podrían relacionarse con la complejidad e inclusividad de las funciones psicológicas.

Dentro de los antecedentes empíricos que se encuentran sobre el tema destaca el trabajo de Serrano (2009), quien realizó una investigación para evaluar la complejidad e inclusividad de las funciones intrasituacionales. Para tales propósitos, el autor expuso a ratas (cepa Wistar) a diferentes situaciones de entrega de agua correspondientes a tres programas de estímulo - contingente, no contingente y contingente condicional- (véase, p. ej., Schoenfeld \& Cole, 1972), en donde, en los subciclos $t^{d}$ de cada programa, la posibilidad de entrega de agua se señalaba con luces led de color rojo o verde relacionadas con distintas condiciones de respuesta, según el programa vigente.

En dicho trabajo, las situaciones suponían la evaluación y entrenamiento de la función contextual, suplementaria y selectora, respectivamente, cada una con una duración de 30 sesiones. En particular, en la situación no contingente la entrega de agua no dependía de la respuesta de la rata, pues se entregaba cada que terminaba la activación de la luz roja, independientemente de cualquier respuesta del sujeto experimental - así, cuando este diferenciaba la relación luz-agua, podía aumentar la frecuencia de consumo de agua-. Por otra parte, en la situación contingente la respuesta a una palanca era necesaria para la entrega de agua, aunque la respuesta solo era efectiva ante la luz roja. Y, por último, en la situación contingente condicional la entrega de agua dependía de la respuesta a una palanca derecha o izquierda, respecto al bebedero, según la ocurrencia de la luz roja o verde; esta situación implicó la respuesta precisa en relación con dos condiciones de respuesta: (a) luz rojarespuesta a palanca izquierda y (b) luz verde-respuesta a palanca derecha (Serrano, 2009).

A partir de esto, todos los sujetos se expusieron a las situaciones experimentales de manera concurrente en un espacio cuadrangular con cuatro paneles de actividad - un bebedero, dos palancas y luces led-; y cada programa de entrega de agua estaba disponible en uno de tres paneles de actividad, quedando el cuarto panel sin consecuencias programadas. De manera general, los resultados de Serrano (2009) mostraron una disminución progresiva en los índices de ajuste - unidad de medida propuesta por el autor - respecto al incremento de la complejidad del comportamiento evaluado y entrenado en las situaciones experimentales; sin embargo, si bien este hallazgo se presentó como evidencia relacionada con la complejidad del criterio de ajuste, destaca el hecho de que los índices logrados por los sujetos en las situaciones de entrega de agua contingente y contingente condicional fueron cercanos a cero. Al parecer, no hubo un efecto consistente relacionado con las manipulaciones, lo cual dificulta el análisis del tránsito entre situaciones experimentales - dato asociado a la complejidad e inclusividad funcional- .

En un trabajo posterior, Serrano (2016) realizó un estudio similar en el que expuso a tres ratas (de cepa Wistar), a una secuencia de 10 sesiones de entrega de agua bajo las condiciones no contingente, contingente y contingente condicional. Respecto al estudio de referencia, se modificó la modalidad de los estímulos de visuales a auditivos y se hicieron precisiones en las fórmulas para obtener índices de ajuste que reflejaran mejor el nivel de interacción evaluado, pero los resultados fueron similares a los de Serrano (2009). En resumen, debido al pobre desempeño que mostraron las ratas en las tareas, tampoco se observó el efecto de facilitación de la historia de interacción en un nivel de aptitud funcional de cierta complejidad sobre el ajuste conductual en otro de mayor o menor complejidad. Además, aunque en ambos estudios disminuyó el índice de ajuste con respecto a la complejidad de la tarea enfrentada, este índice se acercó a cero en las dos tareas de mayor complejidad. 
292

Tomando en cuenta lo anterior, en futuros estudios podría considerarse un nivel de desempeño "óptimo" para transitar a una tarea de mayor o menor complejidad. De adoptar este criterio, se podría observar el efecto de campos o funciones parcialmente ajustadas o ajustadas - como tendencia, no como logro (véase Ribes-Iñesta, 2007) — sobre el proceso de ajuste en otras funciones, en lugar de observar el efecto de la transición entre situaciones independientemente del nivel de ajuste alcanzado.

Por otra parte, Ribes-Iñesta et al. (2009) encontraron resultados que podrían considerarse como evidencia en contra del supuesto de inclusividad. En su estudio, los autores entrenaron a participantes para responder en tareas estructuradas con respecto a los cinco niveles de organización funcional, tras lo cual fueron expuestos a sesiones de preprueba y prueba de cada uno de los niveles funcionales, siendo asignados a uno de seis grupos que diferían según el tipo y la cantidad de sesiones de entrenamiento. Específicamente, el Grupo 1 se expuso a sesiones de entrenamiento de los cinco niveles, el Grupo 2 a cuatro - de la segunda función más compleja a la quinta—, y así hasta llegar al Grupo 6, que no recibió entrenamiento. Como medida conductual se utilizó el porcentaje de aciertos obtenidos por los participantes en las sesiones experimentales. En general, los resultados mostraron que es posible adquirir un desempeño eficaz en un nivel de organización funcional de complejidad mayor, sin entrenamiento explícito en los niveles funcionales que lo anteceden.

Sin embargo, pese a los resultados encontrados hasta el momento, el estado de la investigación en el área apenas se desarrolla, por lo que se plantea la necesidad de diseñar y probar métodos de medición y experimentación que permitan dilucidar la complejidad e inclusividad de cada uno de los niveles de organización funcional propuestos en la taxonomía de Ribes y López (1985).

Ahora bien, tomando en cuenta que la evidencia empírica enfocada en mejorar el entendimiento los supuestos de inclusividad y de complejidad progresiva (Ribes-Iñesta et al., 2009; Serrano, 2008, 2009) es poco robusta, la presente investigación tuvo como propósito la realización de un estudio exploratorio con el cual, por medio de una tarea experimental novedosa, se puedan sumar evidencia al análisis de las funciones intrasituacionales. En específico, se planteó la evaluación del desempeño de participantes universitarios en tres situaciones experimentales que incluían niveles de organización funcional de distinta complejidad, con tareas presentadas en tres secuencias diferentes: ascendente, descendente-ascendente y descendente. Así, con el fin de obtener datos relacionados a la complejidad funcional, se comparó el proceso de aprendizaje en la estructuración de las tres funciones intrasituacionales sin historia de aptitud funcional previa; y, respecto a la inclusividad, se analizó el efecto de la historia de aptitud funcional en transiciones descendentes y ascendentes.

\section{Método}

\section{Diseño}

Se utilizó un diseño experimental multicondicional de medidas repetidas " 3 -entre" (secuencias) y " $4-n$ dentro" (sesiones de entrenamiento), con grupos controles. Las condiciones "entre" fueron: (a) ascendente - contextualsuplementario-selector-, (b) descendente-ascendente - suplementario-contextual-selector-y (c) descendente - selector-suplementario-contextual- En combinación con la condición anterior, se hicieron cuatro sesiones de entrenamiento de respuestas a propiedades de los estímulos, a saber: (a) especie, (b) tamaño, (c) motricidad y (d) todas - especie-tamaño-motricidad-. Esta última manipulación se realizó para entrenar respuestas a propiedades absolutas-constantes y relativas-variantes de los estímulos. A partir de esto, los grupos de control no recibieron entrenamiento, y todos los grupos, conformados por cuatro participantes, fueron expuestos a una sesión de preprueba y a una o varias sesiones de prueba según cada nivel de organización funcional. Solo los participantes de los grupos experimentales podían enfrentar en más de una ocasión las sesiones de entrenamiento o prueba.

Para avanzar a la siguiente sesión era necesario que el participante obtuviera un índice de ajuste (IA) igual o mayor a 0.8 en el arreglo contingencial, unidad de medida que se describirá más adelante. Cuando el participante no obtenía un IA igual o superior a 0.8 en una sesión de entrenamiento, volvía a enfrentar la misma sesión hasta alcanzar dicho índice; y, si no lo lograba alcanzar en alguna sesión de prueba, volvía a enfrentar la última sesión de entrenamiento para realizar nuevamente la sesión de prueba. El máximo de oportunidades para alcanzar el IA de 0.8 fue de tres sesiones; al no satisfacer ese criterio en la tercera oportunidad, se descartaba al participante del estudio, agradeciendo su participación.

Ahora bien, hubo cuatro tipos de sesiones de entrenamiento con respecto al tipo de propiedad del estímulo al que se relacionaba la respuesta durante la interacción, a saber: (a) especie, (b) tamaño, (c) locomoción y (d) todas - especie-tamaño-locomoción-. En todas las situaciones experimentales se incluyeron estos cuatro tipos de entrenamiento, donde se expuso a los participantes a cada uno de los tipos de entrenamiento en el orden en el que fueron descritos. Una vez que el participante satisfacía el 
criterio de desempeño en la sesión (IA $\geq 0.8$ ), pasaba a la siguiente sesión — si era de entrenamiento- 0 a la siguiente situación experimental — si la sesión era de prueba—; en el caso de ser el tercer arreglo contingencial, culminaba el experimento (véase Tabla 1).

\section{Participantes}

Por medio de un muestreo no probabilístico se seleccionó a 24 estudiantes universitarios de la carrera de psicología -3 hombres y 21 mujeres con edad promedio de 22 años-, pertenecientes a distintas instituciones educativas de la Zona Metropolitana de Guadalajara (México), una pública y dos privadas. Todos los participantes carecían de experiencia en la tarea experimental y fueron contactados por medio de sus profesores, quienes los incentivaron con créditos en sus asignaturas por participar en el experimento.

\section{Aparatos, equipamiento y situación experimental}

Se utilizaron cuatro cubículos semiaislados de ruido y aislados de estímulos visuales ajenos a la tarea experimental. Al interior de los cubículos se encontraba una silla y una mesa con un equipo de cómputo marca HP con ambiente Windows XP, un monitor cromático para la presentación de las tareas experimentales y un mouse para responder a los estímulos. Para la programación de las tareas experimentales y el registro de respuestas se utilizó el programa SuperLab 2.0.4@.

\section{Tareas experimentales}

Según el nivel de organización funcional a medir y entrenar, se utilizó una tarea experimental distinta en cada caso para posibilitar la situación de interacción; y, como estímulos, se utilizaron dibujos de animales en blanco y negro, que variaban en: (a) especie - perro, toro, elefante, picaflor o colibrí, gaviota, águila, atún, delfín y ballena-, (b) tamaño — chico [perro, colibrí y atún], mediano [toro, gaviota y delfín] y grande [elefante, águila y ballena] - y (c) locomoción predominante - caminar [perro, toro y elefante], volar [colibrí, gaviota y águila] y nadar [atún, delfín y ballena] -

También, se utilizaron símbolos encerrados en círculos de distinto color que se relacionaban a una de las propiedades de los dibujos: los de color azul se asociaban a la propiedad especie, representada en los dibujos; los de color verde se asociaban a la propiedad locomoción — caminar, volar y nadar-; y los de color amarillo se asociaron con la propiedad tamaño — chico, mediano y grande - , relativa al grupo de locomoción al que pertenecía cada especie. Cuando en el arreglo de estímulos del ensayo no había ningún símbolo que se relacionara con el dibujo, un círculo con la palabra "ninguna" daba la opción de "omitir la respuesta". Cabe mencionar que en la mayoría de los ensayos la respuesta correcta era por comisión y solo en algunos por omisión, por lo que la opción de respuesta por omisión podía estar presente en los ensayos en los cuales era pertinente emitir u omitir la respuesta (véase Tabla 2).

Ahora, para el arreglo contingencial de tipo contextual se comenzaba cada ensayo con la presentación, durante dos segundos, de uno de los símbolos en la parte izquierda de una pantalla blanca; posteriormente, aparecía durante tres segundos una flecha al lado derecho del símbolo, seguida del dibujo que le correspondía según la fase de entrenamiento

Tabla 1.

Diseño experimental

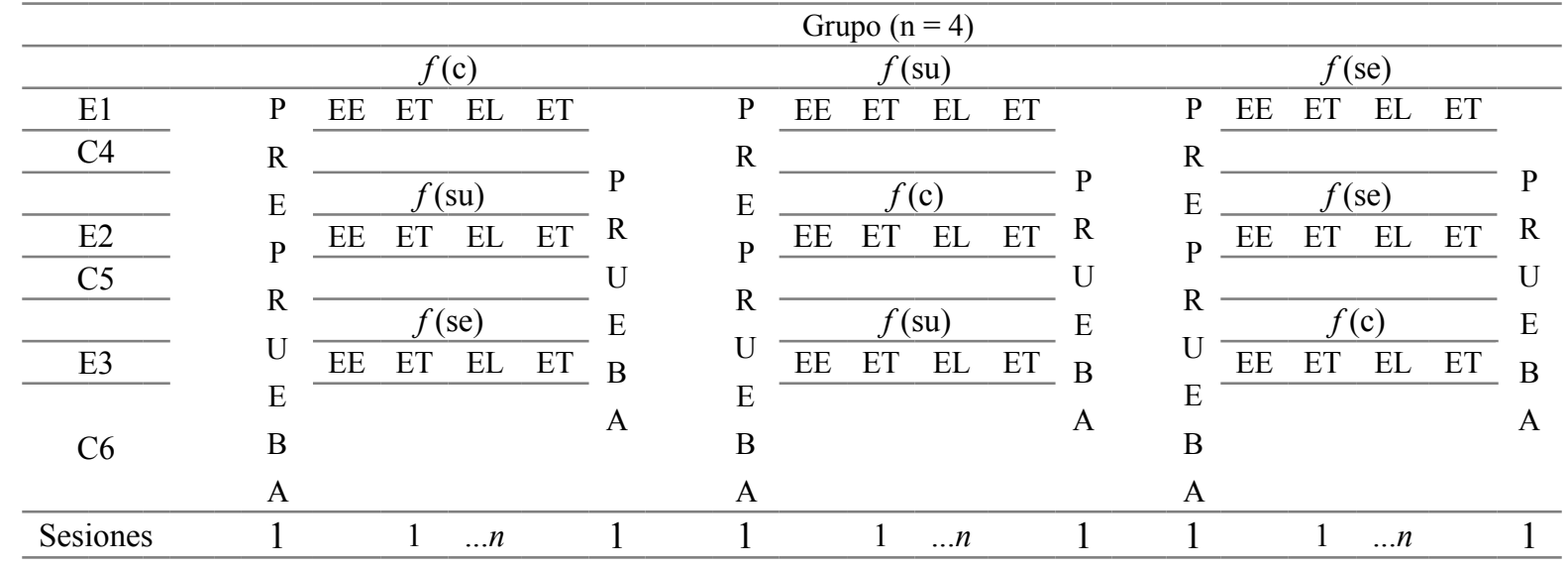

Notas. $\mathrm{E}=$ grupo experimental; $\mathrm{C}=$ grupo control; $f(\mathrm{c})=$ función contextual; $f(\mathrm{su})=$ función suplementaria; $f(\mathrm{se})=$ función selectora; $\mathrm{EE}=$ entrenamiento especie; $\mathrm{ET}=$ entrenamiento tamaño; $\mathrm{EL}=$ entrenamiento locomoción; $\mathrm{ET}=$ entrenamiento todas (todas las propiedades entrenadas del dibujo). 
Tabla 2.

Matriz de estímulos en la que se muestran los símbolos a los que se asoció cada dibujo

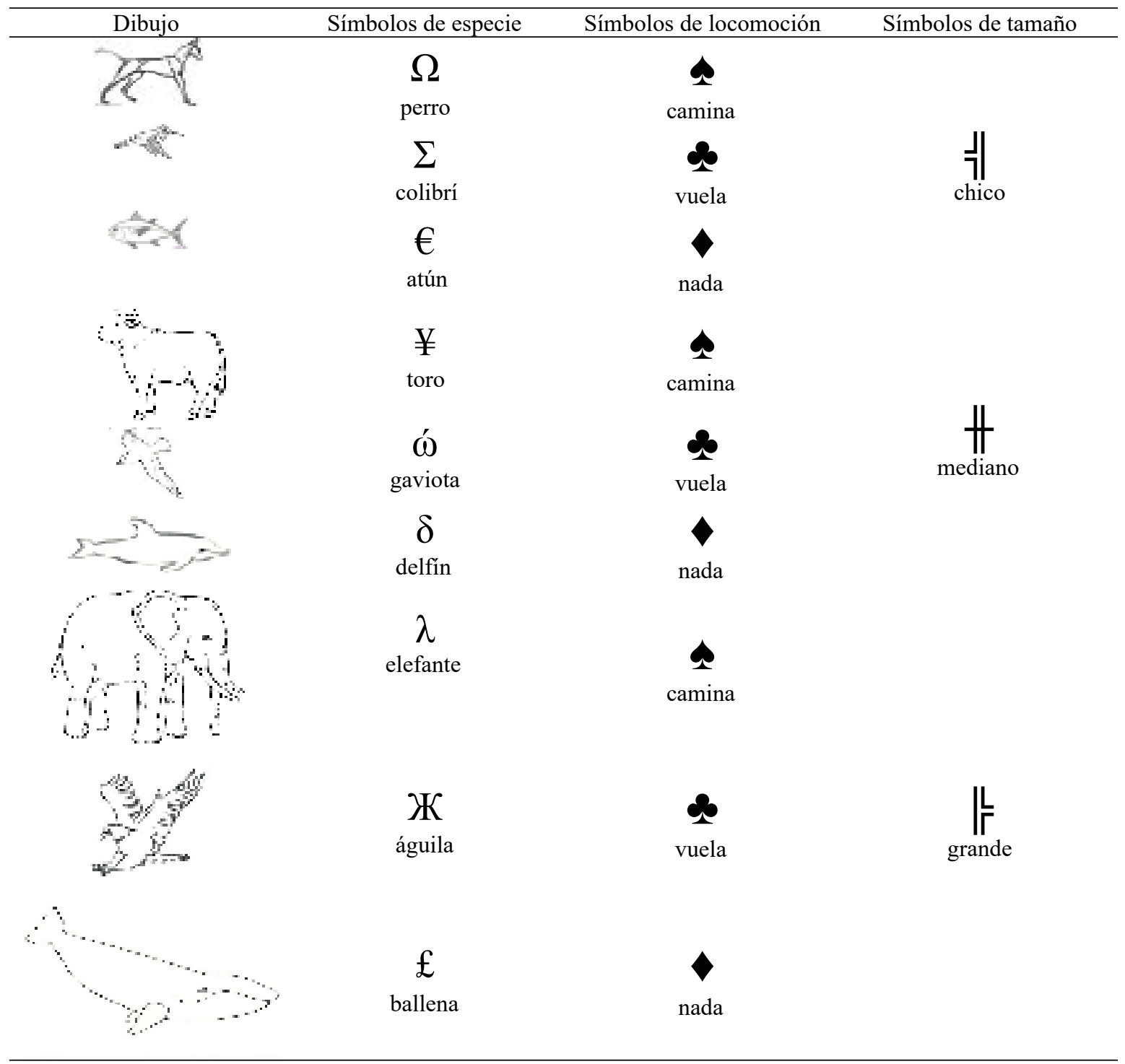

Nota. Círculos de diferentes colores contenían los símbolos para facilitar su discriminación. Los colores azul, verde y amarillo se asociaron con las propiedades de especie, locomoción y tamaño, respectivamente.

o prueba; y, al final, se añadía, durante dos segundos, una segunda flecha seguida de un recuadro que encerraba una palabra en español asociada a una propiedad del dibujo - especie, tamaño o tipo de locomoción predominante(véase Figura 1). Entre ensayos se presentó, durante medio segundo, una pantalla en color negro, a la que se agregaba de manera intermitente una leyenda que decía: "Recuerda, es importante poner atención".

Además de esto, las sesiones de entrenamiento estaban compuestas por dos subsesiones: una de entrenamiento observacional - ensayos previamente descritos - y otra de evaluación. Específicamente, el arreglo de estímulos de los ensayos de evaluación se organizó de manera horizontal, con un símbolo en la parte izquierda de la pantalla y tres recuadros en la parte derecha con palabras dentro - el fondo de la pantalla era blanco- (véase Figura 2). La ocurrencia de ambos tipos de ensayos se presentó de manera aleatoria, y en algunos ensayos de la subsesión de evaluación aparecía un recuadro con la palabra "ninguna". 


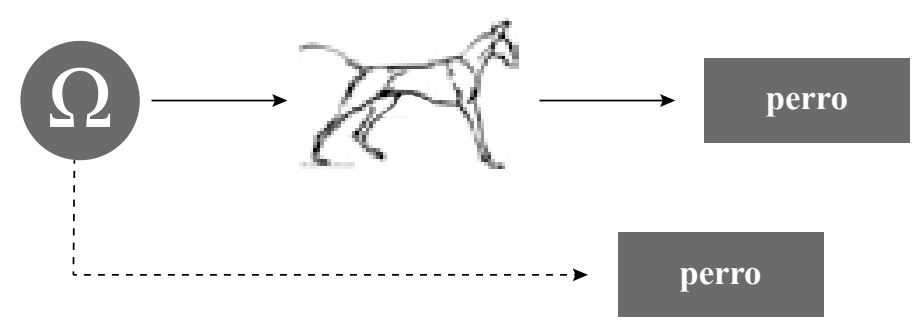

Figura 1. Ensayo de arreglo contingencial de tipo contextual, subsesión de entrenamiento observacional.

En cuanto al procedimiento, (a) la preprueba y la prueba eran idénticas a la subsesión de entrenamiento observacional, en la que se evaluaba la exposición a los ensayos observacionales; (b) no se retroalimentó la ejecución de los participantes en ninguna de las sesiones de prueba; y (c) el número de ensayos por cada sesión varió según el tipo de entrenamiento o prueba - preprueba, entrenamiento y prueba-.

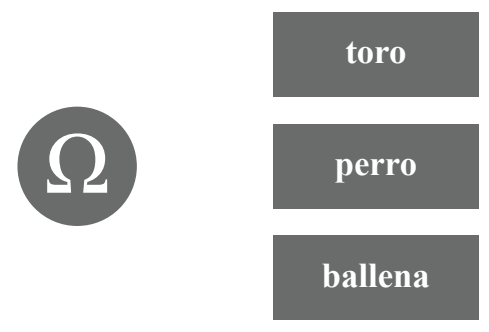

Figura 2. Ensayo de arreglo contingencial de tipo contextual en la subsesión de evaluación —entrenamiento, preprueba y prueba-.

Por otra parte, en el arreglo contingencial de tipo suplementario iniciaba cada uno de los ensayos con la presentación, en una pantalla blanca, de tres círculos alineados verticalmente en la parte izquierda. De entre los tres círculos, solo uno de ellos contenía un símbolo, mientras que los otros dos encerraban la palabra "ninguna" y una o dos letras del alfabeto con la finalidad de hacer "saliente" al símbolo. Si el participante respondía —-"dando clic"—al círculo con un símbolo, una flecha contigua a este aparecía al lado derecho del símbolo, seguida de un dibujo; si respondía al círculo con la palabra "ninguna" cuando los otros dos círculos contenían letras, aparecía durante dos segundos en una nueva pantalla la palabra "correcto"; y cuando respondía a los círculos que contenían letras o al que contenía la palabra "ninguna" cuando en ese ensayo había otro círculo con un símbolo dentro, aparecía durante dos segundos en una nueva pantalla la afirmación "respuesta incorrecta". Después de la retroalimentación comenzaba un nuevo ensayo, y la sesión culminaba hasta que el participante había sido expuesto dos veces a cada uno de los símbolos correspondientes a la sesión de entrenamiento.

Debido a que en la situación de tipo suplementario se entrenaba al participante a responder al símbolo para suplementar su relación con un dibujo, sin asegurar que estaba diferenciando las relaciones que suplementaba, en las pruebas ya no se presentaban letras, sino únicamente símbolos o, en algunos ensayos, un círculo con la palabra "ninguna". Por otra parte, el arreglo de estímulos era similar al del entrenamiento, pero con un dibujo en la parte derecha de la pantalla; y, de entre los tres símbolos, solo uno de ellos correspondía al dibujo. Si se respondía a este aparecía una flecha entre el símbolo y el dibujo; y cuando no era así, comenzaba un nuevo ensayo (véase Figura 3). Cabe mencionar que la preprueba y la prueba se diseñaron
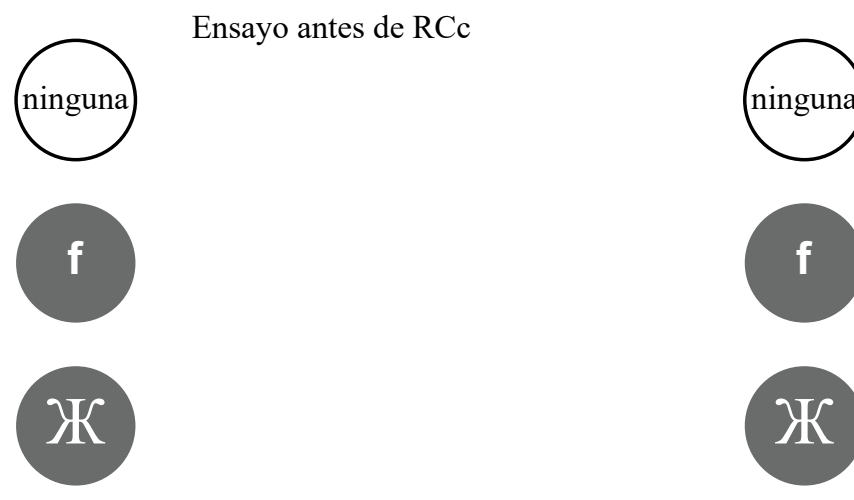

Ensayo después de RCc

Figura 3. Ensayo del arreglo contingencial de tipo suplementario. 
de esta manera para evaluar si la ejecución de cada uno de los participantes implicaba la diferenciación de cada una de las funciones de los símbolos respecto a los dibujos que se presentaban; reactividad necesaria para responder de manera efectiva.

Finalmente, para la evaluación del ajuste preciso en la condición selectora, la tarea experimental tuvo un arreglo contingencial típico de igualación de la muestra de primer orden, en donde cada ensayo contuvo, de manera simultánea, en la parte superior de la pantalla un dibujo y en la parte inferior cuatro círculos. Casi todos los círculos contenían símbolos, con excepción de unos cuantos ensayos en los que se presentó en el interior de uno de ellos la palabra "ninguna".

Durante las sesiones de entrenamiento, los participantes recibían retroalimentación continua para cada una de sus elecciones en ensayos correctivos. Este cambio en el procedimiento respecto a las tareas previas se hizo porque cuando se piloteó la tarea sin ensayos correctivos los participantes requerían de más sesiones para alcanzar el criterio de ajuste. Respecto a la parte cualitativa, la tarea se complejizaba porque un mismo dibujo se relacionaba con distintos símbolos según el momento de respuesta; así, la pertinencia de la respuesta cambiaba de un ensayo a otro según el arreglo de estímulos presentado, por lo que mientras que en un ensayo era pertinente responder al tamaño, en otro lo era su locomoción o la especie de animal representada en el dibujo (véase Figura 4).

En este arreglo contingencial de tipo selector, si se respondía a los círculos que contenían el símbolo correspondiente al dibujo o a la palabra "ninguna" cuando no había un símbolo correspondiente, aparecía en una nueva pantalla la palabra "correcto" durante dos segundos, y posteriormente iniciaba un nuevo ensayo; pero si se respondía a los círculos con un símbolo que no correspondía al dibujo o al que contenía la palabra "ninguna" cuando en el ensayo había un símbolo correspondiente, aparecía la afirmación "respuesta incorrecta" durante dos segundos y se repetía el ensayo en el que se había errado hasta lograr una respuesta correcta. No se retroalimentó la ejecución en la preprueba ni en la prueba.

Es importante mencionar que para evaluar el desempeño de los participantes en las tareas experimentales se utilizó el indice de ajuste (IA) como unidad de medida, el cual muestra la tendencia de los participantes a ajustar su respuesta a las circunstancias de cada tarea en una sesión, por medio de la siguiente fórmula:

$$
\mathrm{IA}=\frac{(\mathrm{RC}-\mathrm{RI})}{\mathrm{RT}}
$$

Específicamente, el IA resulta de la sustracción de las respuestas correctas (RC) menos las respuestas incorrectas (RI), entre las respuestas totales (RT) programadas en una sesión, equivalente al número de ensayos. Aunque cabe mencionar que en algunos ensayos de las tareas experimentales se dio la opción de no responder cuando ninguna de las opciones de respuesta era pertinente, de manera que era posible emitir RC por comisión y por omisión, así como RI por comisión y omisión. Asimismo, se consideró RC por comisión la respuesta a estímulos a los que era pertinente responder en un ensayo, y RI por comisión a la respuesta a estímulos a los que no era pertinente responder; en algunos ensayos se daba la opción de "no responder", al seleccionar la opción de respuesta "ninguna" — que se presentaba entre los estímulos-; si se optaba por "no responder" cuando en el ensayo no había algún estímulo al que fuera pertinente responder, la respuesta se consideró RC por omisión. Por último, las RI por omisión eran aquellas en las se optaba por "no responder" cuando en el ensayo sí había un estímulo al que era pertinente responder.
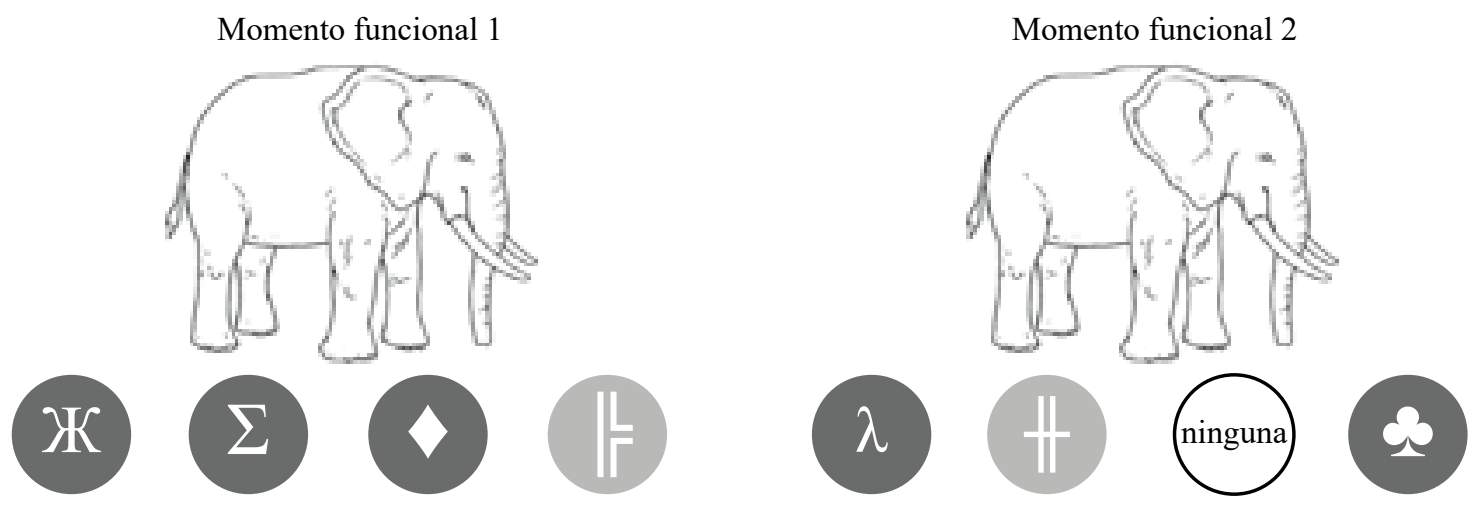

Figura 4. Ensayo del arreglo contingencial de tipo selector. En el momento funcional 1 la respuesta pertinente es el símbolo “怰”, y, en el momento funcional 2 , el símbolo “ $\lambda$ ”. 
Finalmente, se consideró que la particularidad del ajuste - diferencial, efectivo o preciso- derivaba del arreglo contingencial en el que el participante respondía - contextual, suplementario y selector, respectivamente-

\section{Procedimiento}

El estudio daba inicio con el ingreso de los participantes a los cubículos experimentales, en donde se les explicaba que la investigación se enfocaba al estudio del aprendizaje. Posteriormente, se les entregaba la carta de consentimiento informado, y, si estaban de acuerdo, la firmaban y se daban las instrucciones pertinentes para responder en los arreglos contingenciales. En este momento, el experimentador comentaba que las instrucciones se presentarían en el monitor, que tenían que responder manipulando el "mouse" — dando clic al botón izquierdo de este- $-y$ que la duración del experimento podría ser de dos horas aproximadamente. Después de haber entregado las anteriores aclaraciones, el encargado del experimento se retiraba del cubículo y cerraba la puerta.

Cada sesión experimental presentaba en la primera diapositiva de inicio de la sesión los datos institucionales del centro de investigación donde se llevó a cabo el estudio, y en la siguiente pantalla se daba la bienvenida a la sesión y se especificaba el arreglo contingencial que iba a enfrentar el participante preprueba, entrenamiento o prueba - para evitar que la respuesta se pudiese afectar por la similitud de contextos sin ninguna señal agregada que permitiera diferenciarlos; dicho efecto se encontró en algunos estudios que utilizan tareas de igualación a la muestra de primer orden (p. ej., González \& Ortiz, 2014). Adicional a esto, en la misma diapositiva aparecía una instrucción general que variaba según la sesión en la que se encontraba el participante (véase Tabla 3).

En lo que respecta a los participantes de los grupos controles, estos se expusieron a una sesión de preprueba y prueba de las tres tareas experimentales, donde varió la secuencia de exposición con respecto al grupo asignado; además, ninguno de ellos fue expuesto a sesiones de entrenamiento, por lo cual culminaban su participación en el estudio después de aproximadamente 50 minutos.

Por otra parte, debido a la naturaleza de cada una de las funciones psicológicas estudiadas, la forma de entrenar y evaluar no solo varió en cuanto al arreglo de estímulos, sino también respecto al número de ensayos de cada tarea experimental. Sin embargo, se buscó homogenizar el número de ensayos en las sesiones de preprueba y prueba con 18 ensayos, nueve para evaluar la relación con la propiedad especie, tres con locomoción, tres con tamaño y tres ensayos con omisión de respuesta. Además de esto, el entrenamiento del ajuste diferencial de especie, tamaño y locomoción constaba de 10 ensayos, nueve para relacionar los símbolos con cada dibujo y uno para omitir respuesta; y, por último, en la sesión en la que se entrenaban todas las funciones se utilizaron 18 ensayos, nueve para asociar los símbolos con la propiedad especie, tres con locomoción, tres con tamaño y tres con la omisión de respuesta.

Asimismo, el ajuste efectivo y preciso se entrenaba en 21 ensayos respecto a la especie representada en los dibujos, 18 para relacionar cada símbolo dos veces con su dibujo correspondiente y tres para omitir respuesta; mientras que el entrenamiento de la repuesta al tamaño o locomoción constaba de 27 ensayos. Por otra parte, cada símbolo se asociaba dos veces con cada dibujo respecto a su tamaño o locomoción, $\mathrm{y}$, además, se relacionaba dos veces con el grupo de dibujos al que correspondían —es decir, en un mismo ensayo aparecía el atún, el delfín y la ballena con asociación al rombo/diamante-, esto sumado a tres ensayos para omitir respuesta.

Cuando se entrenó la respuesta efectiva respecto a todas las propiedades de los estímulos (especie-locomocióntamaño) se requirió de 36 ensayos, 18 para la propiedad especie, seis para locomoción, seis para tamaño y seis para omitir respuesta. Respecto a esto, cabe resaltar que hubo un incremento considerable en el número de ensayos en el entrenamiento del ajuste preciso de la respuesta a todas las propiedades del dibujo, con un total de 76: 18 relacionadas con la especie, 24 con el tamaño, 24 con la locomoción y 10 para omitir respuesta. De los 24 ensayos de respuesta al tamaño y locomoción, 18 fueron para relaciones uno a uno, y seis para relaciones uno a muchos, con un símbolo-un dibujo o un símbolo-tres dibujos, respectivamente.

Finalmente, el entrenamiento se dividió en cuatro fases debido a que el arreglo contingencial de ajuste preciso en los que se presentaron todas las categorías resultó ser muy complejo. Esto se tomó en cuenta debido a que, en una prueba piloto de dicho procedimiento, de 10 participantes ninguno pudo mostrar un índice de ajuste cercano a 0.8 después de cuatro sesiones de 76 ensayos a pesar de que el procedimiento era correctivo. Por ello, se decidió segmentar el entrenamiento en cuatro fases, y, para que cada uno de los arreglos contingenciales fuera comparable, los arreglos de menor complejidad también tuvieron cuatro fases - contextual y suplementario, aunque se redujo el número de ensayos según la complejidad funcional para disminuir el tiempo del experimento y reducir la probabilidad de que la fatiga pudiera interferir en los resultados.

\section{Análisis de datos}

Se utilizó la prueba U de Mann-Whitney para analizar las diferencias en los resultados de los participantes de los Grupos 1, 2 y 3 en la primera tarea que enfrentaron; tareas 


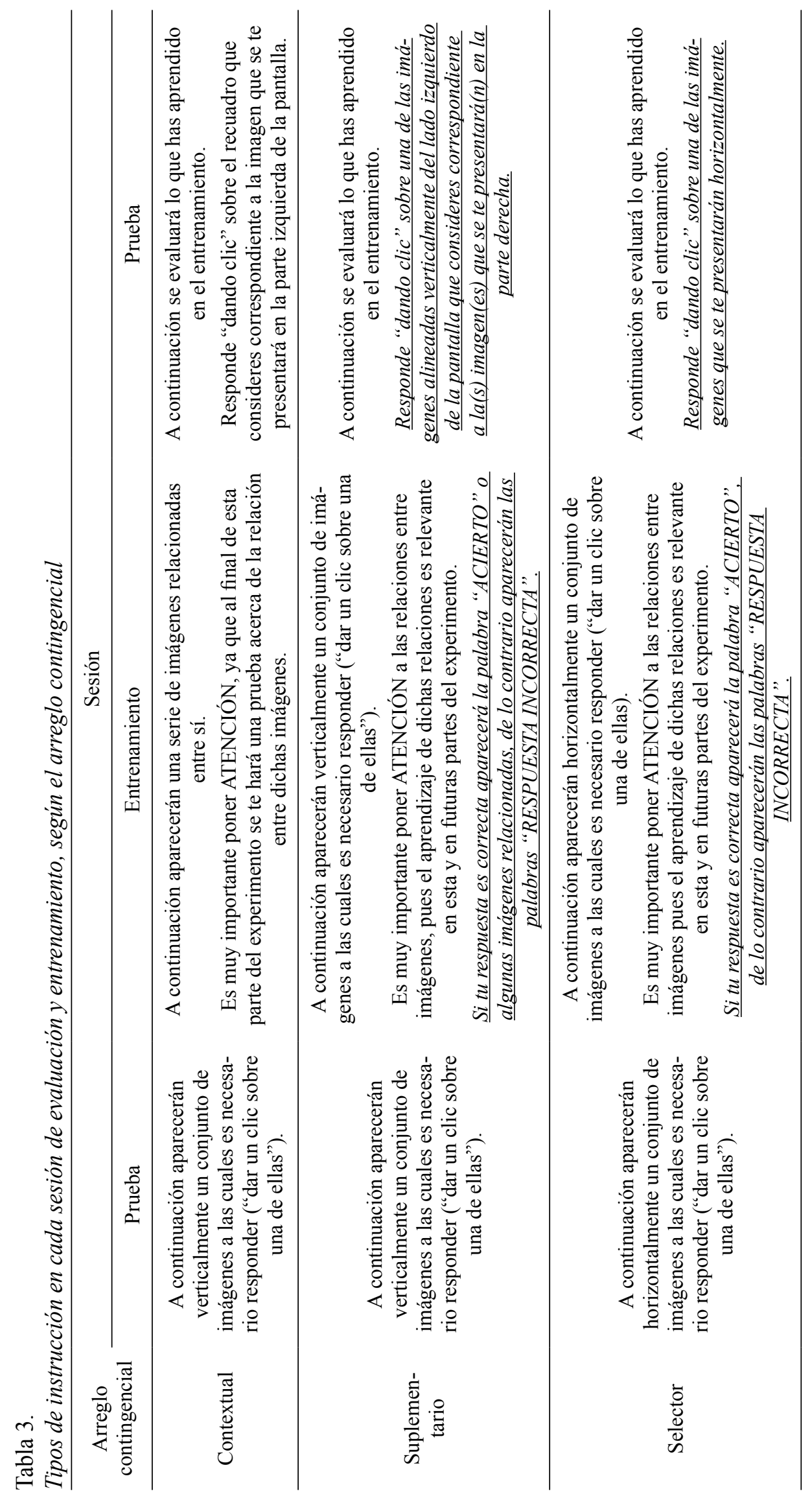


que variaban en grado de complejidad. El dato que se analizó fue el número de sesiones necesarias para alcanzar el criterio de ajuste en la prueba.

\section{Resultados}

El dato que se graficó para el análisis de los resultados fue el indice de ajuste (IA) que los participantes obtuvieron en cada una de las sesiones de las fases experimentales de los arreglos contingenciales a los que se expusieron. Así, los resultados de cada grupo de participantes se organizaron en figuras con 12 gráficos —en el eje de las ordenadas el IA obtenido y en el eje de las abscisas el número de sesiones - ordenados en tres columnas y cuatro hileras que corresponden a la ejecución de un participante en diferentes arreglos contingenciales - en cada columna se muestra la ejecución de los participantes del mismo grupo en un arreglo contingencial-; donde se diferencian las figuras según la secuencia de exposición a los arreglos contingenciales, representando la ejecución de un grupo de participantes.

La representación del IA mostrado por los participantes en cada sesión se graficó de la siguiente manera: (a) una barra en color gris colocada en el extremo izquierdo del gráfico para la preprueba; (b) una o varias barras en color gris en el extremo derecho de la gráfica para la prueba dependiendo de si se había cumplido o no con el criterio de ajuste funcional, cada barra representa una exposición a la sesión de prueba-; y (c) círculos y cuadrados aislados o unidos por líneas para el entrenamiento - se unen con una línea cuando el participante tenía que exponerse a más de una sesión por no haber cumplido con el criterio de ajuste en dicha sesión-. Adicionalmente, de izquierda a derecha, el primer círculo corresponde a la ejecución en "entrenamiento especie", el primer cuadrado representa el "entrenamiento tamaño", el segundo círculo, el "entrenamiento locomoción", y el segundo cuadrado, el "entrenamiento todos" - especie-tamaño-locomoción-.

Sumado a lo anterior, los gráficos presentan una línea segmentada en posición horizontal que indica el criterio de ajuste funcional $(\geq 0.8)$ para el avance a la siguiente sesión; en algunos casos se encuentra una cifra menor a -0.2 para indicar el IA que no pudo ser representado en la gráfica con una barra de mayor longitud; $y$, por último, en cuanto a la descripción genérica de cada uno de los gráficos, en la parte superior de las columnas de los gráficos se indica el tipo de arreglo contingencial por medio de un rótulo. En particular, el rótulo $f(\mathrm{CO})$ indica la columna de gráficas que representan el IA diferencial en el arreglo contingencial de la función contextual, el $f(\mathrm{SU})$ la del IA efectivo de la función suplementaria, y el $f(\mathrm{SE})$ la del IA preciso de la función selectora.

Teniendo esto en cuenta, en la Figura 5 se encuentran los resultados del Grupo E1 (contextual-suplementario-selector), donde se puede ver que los cuatro participantes obtuvieron un IA diferencial menor o cercano a cero en la preprueba; en el entrenamiento, tres participantes lograron, en su primer intento, cumplir con el criterio de ajuste funcional en cada una de las sesiones, así como en la prueba — solo el participante P2 requirió de cuatro sesiones en "entrenamiento especie" y tres sesiones en "entrenamiento locomoción" para cumplir con el criterio de ajuste funcional, sumando 11 sesiones en el arreglo contingencial (AC) contextual-; y en cuanto a la ejecución de los participantes en los AC suplementario y selector, se encontró que en las prepruebas y pruebas casi todos rebasaron el valor de 0.8 de IA en una sesión - solo los participantes P1 y $\mathrm{P} 2$ tuvieron que enfrentar más de seis sesiones en alguno de los AC posteriores al de tipo contextual-.

Por otra parte, en la Figura 6 se muestran los índices de las ejecuciones de los participantes del Grupo E2, quienes enfrentaron primero el AC suplementario. En general, se encontró que los cuatro participantes obtuvieron un IA cercano a cero en la primera preprueba que enfrentaron, dato similar al de los participantes del Grupo E1. Sin embargo, tres de los cuatro participantes mostraron diferencias considerables en cuanto el incremento del número de sesiones requeridas para cumplir con el criterio de ajuste en las sesiones de entrenamiento o de prueba - P6 con 9 sesiones, $\mathrm{P} 7$ con 12 sesiones y P8 con 9 sesiones-. Finalmente, con respecto a los IA obtenidos en los dos siguientes AC (contextual y selector), se encontró que casi todos los participantes requirieron el mínimo de sesiones para la conclusión de todas las fases experimentales, con excepción del participante P7, quien requirió de 7 sesiones en el AC contextual.

De igual forma, la ejecución de los participantes del Grupo E3 se muestra en la Figura 7, en donde destaca que, respecto a la ejecución en los arreglos contingenciales en los que los participantes carecían de historia de aptitud funcional se requirió de más sesiones - entre 10 y 15 para lograr el criterio de ajuste preciso en las sesiones de entrenamiento y prueba del AC selector. Además, los participantes tuvieron que exponerse dos o más veces a casi todas las sesiones para lograr el criterio de ajuste, efecto que fue más agudo en dos participantes que se expusieron a más de una sesión de prueba (P9 y P12). En cuanto al dato obtenido en la preprueba y prueba de los siguientes $\mathrm{AC}$ - en este caso, suplementario y contextual-, se puede observar que se requirió el mínimo de sesiones para cumplir cada tarea, excepto para $\mathrm{P} 10$ y $\mathrm{P} 12$, quienes requirieron de una sesión más (7 sesiones) en el AC suplementario. 
$\mathrm{f}(\mathrm{CO})$
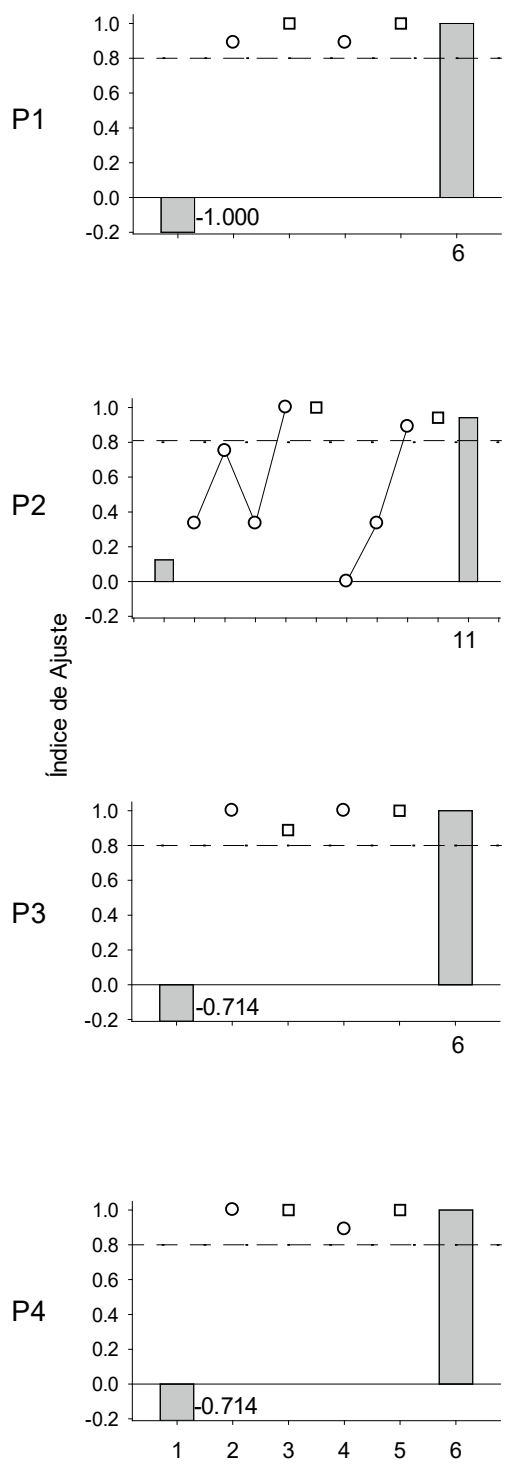

$\mathrm{f}(\mathrm{SU})$
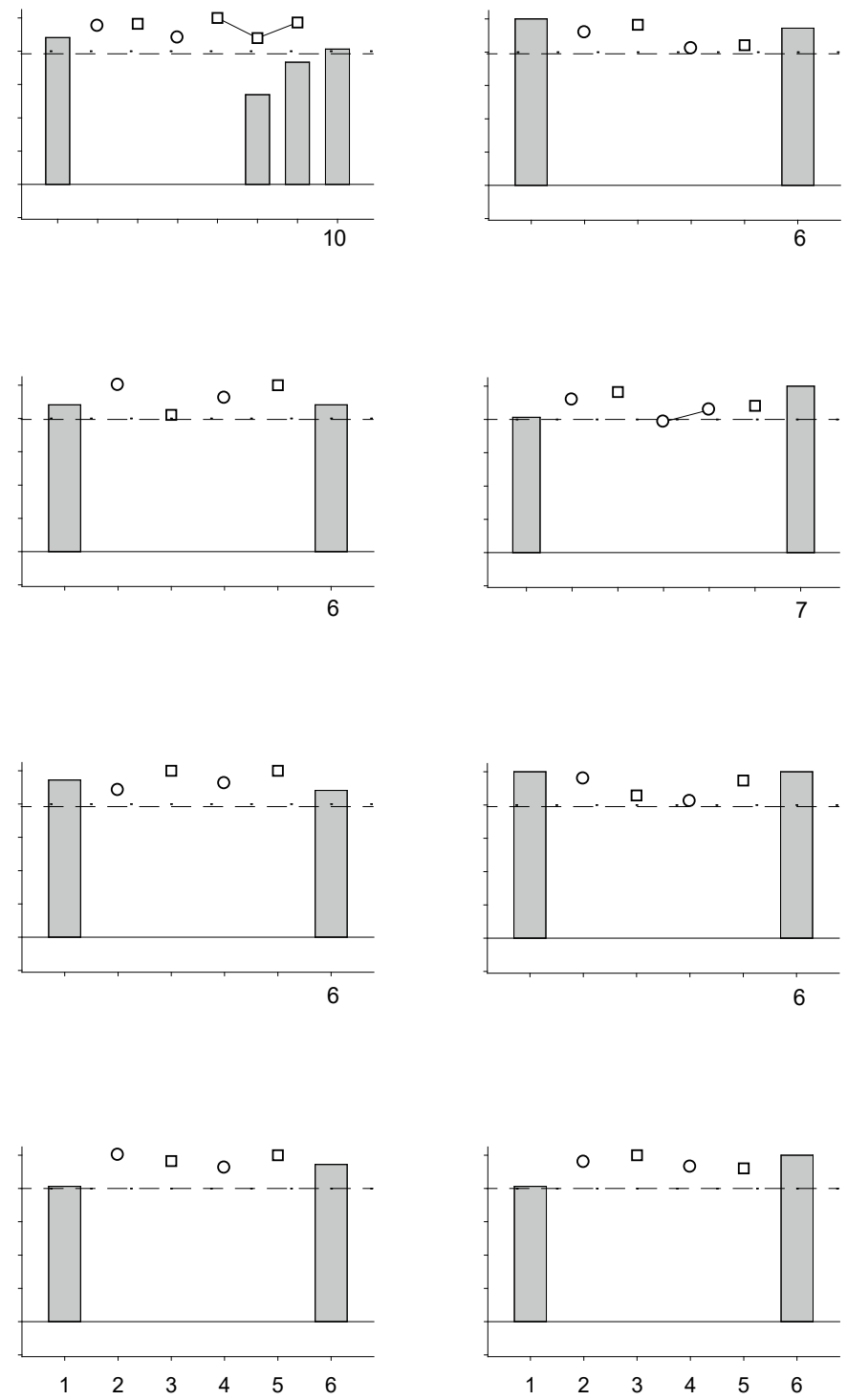

Figura 5. Índices de ajuste logrados por los participantes del Grupo E1. $f(\mathrm{C})=$ función contextual, $f(\mathrm{SU})=$ función suplementaria, $f(\mathrm{SE})=$ función selectora, $\mathrm{P}=$ participante. Las barras muestran la ejecución en preprueba y prueba — de izquierda a derecha, respectivamente-; los círculos y cuadrados representan la ejecución en las sesiones de entrenamiento; y la línea horizontal discontinua ubicada en el índice de ajuste de 0.8 indica el criterio de ajuste para pasar a la siguiente sesión. 
$\mathrm{f}(\mathrm{SU})$
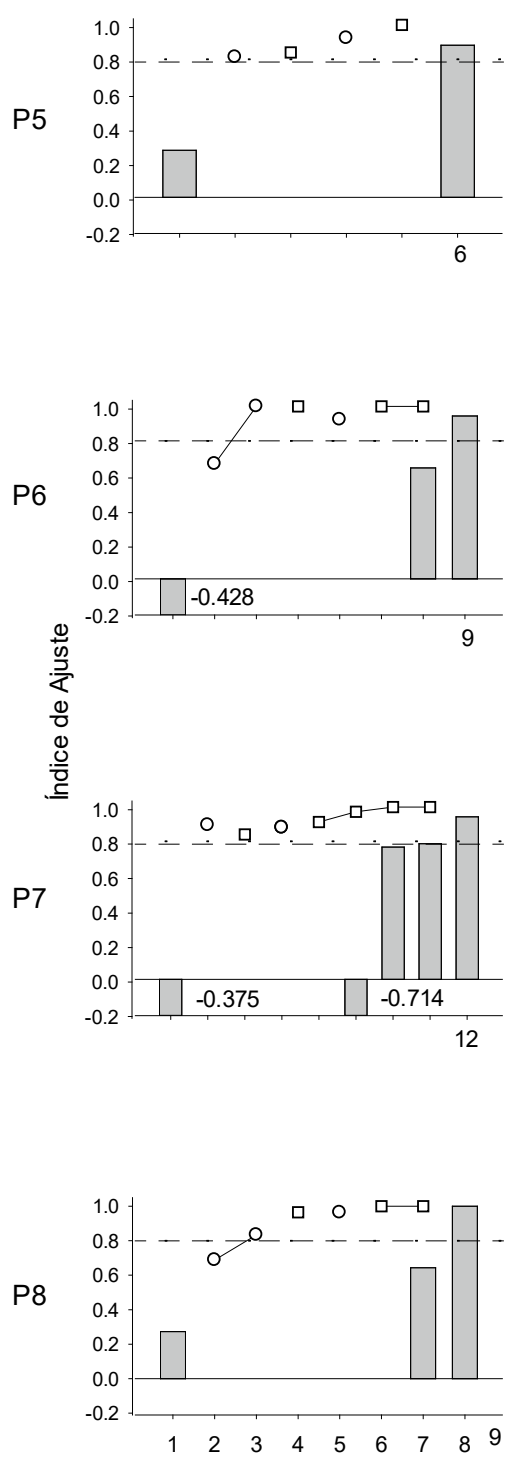

$\mathrm{f}(\mathrm{CO})$
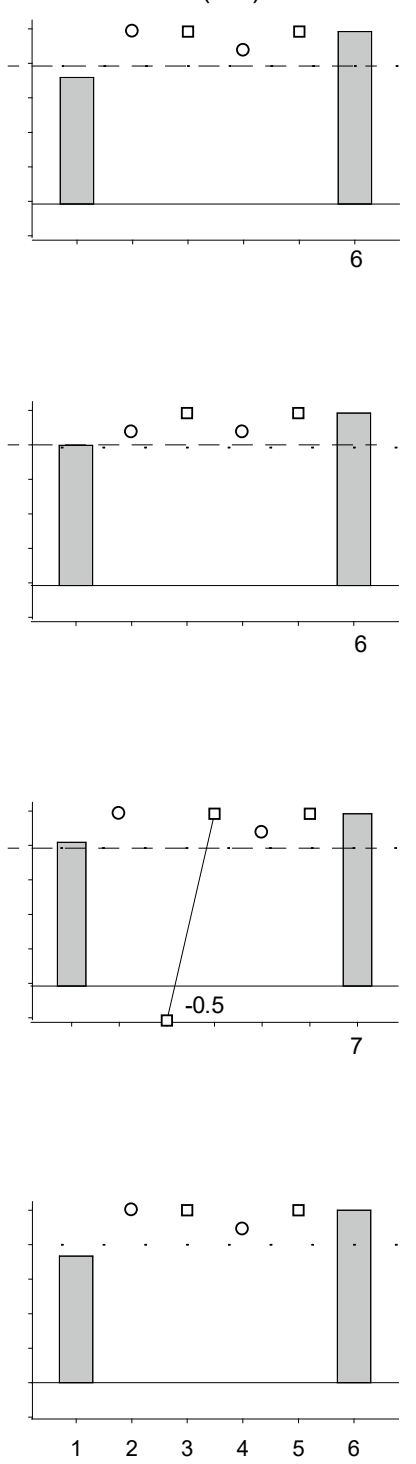

f(SE)
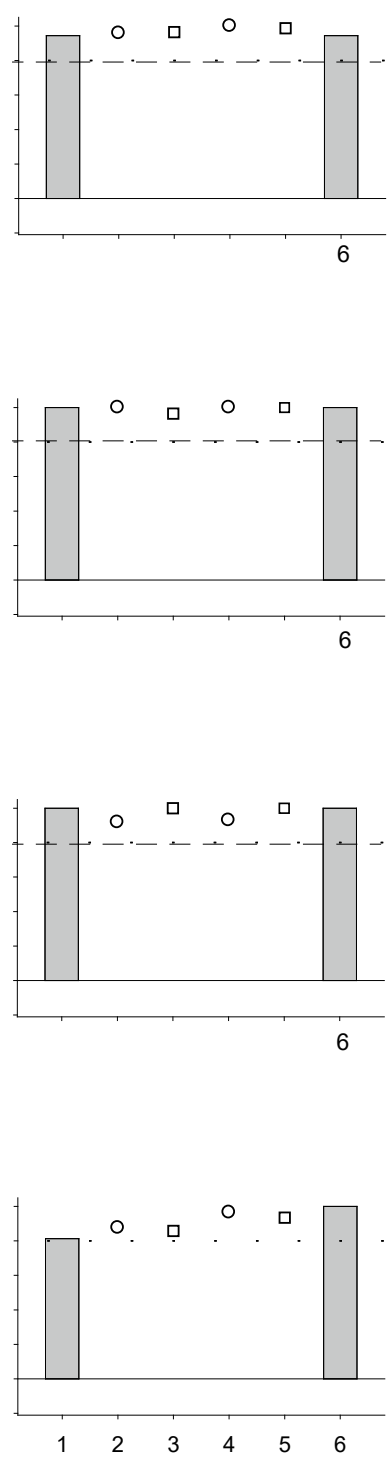

Sesiones

Figura 6. Índices de ajuste logrados por los participantes del Grupo E2. $f(\mathrm{C})=$ función contextual, $f(\mathrm{SU})=$ función suplementaria, $f(\mathrm{SE})=$ función selectora, $\mathrm{P}$ = participante. Las barras muestran la ejecución en preprueba y prueba — de izquierda a derecha, respectivamente-; los círculos y cuadrados representan la ejecución en las sesiones de entrenamiento; y la línea horizontal discontinua ubicada en el índice de ajuste de 0.8 indica el criterio de ajuste para pasar a la siguiente sesión. 

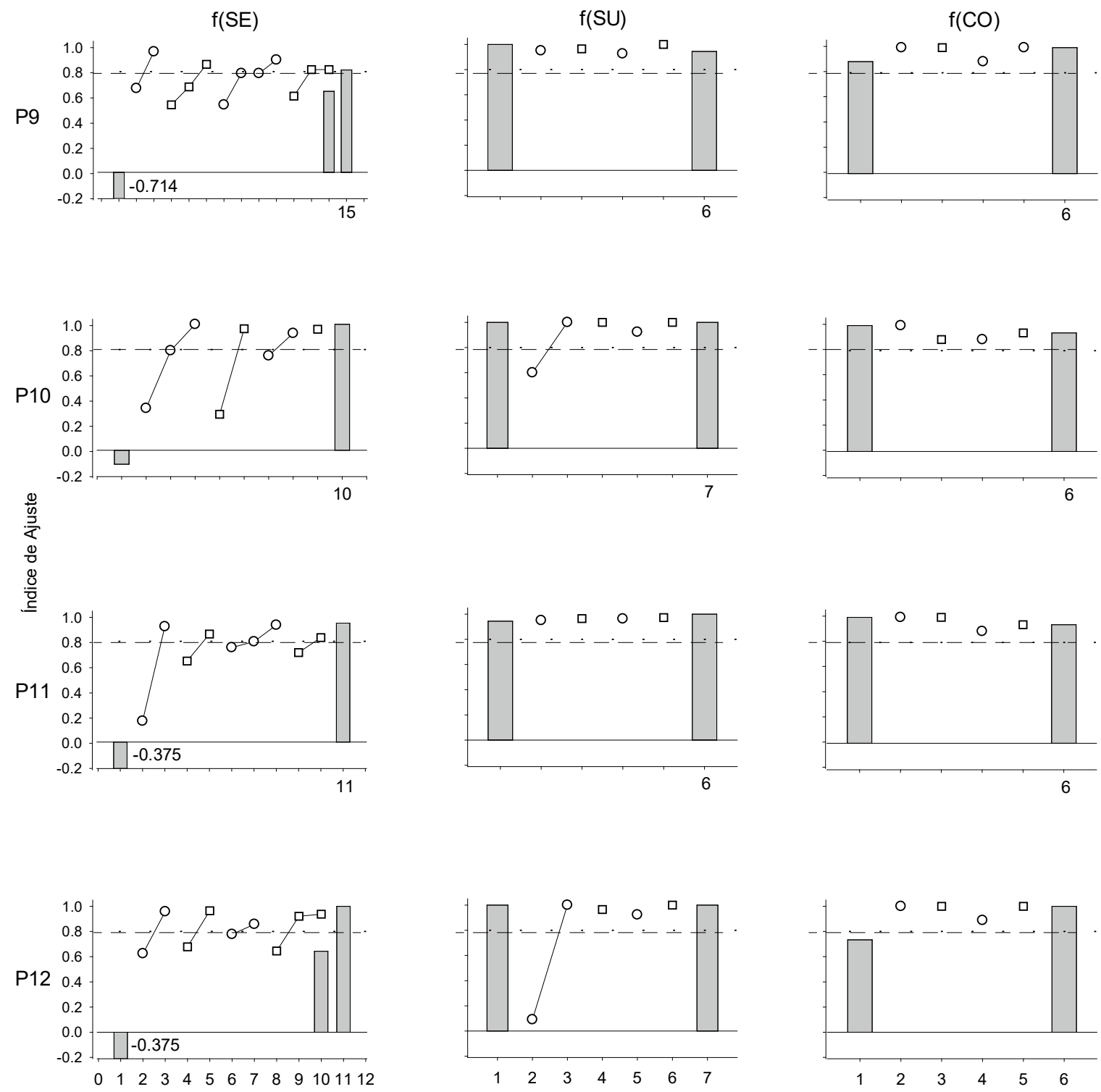

Sesiones

Figura 7. Índices de ajuste logrados por los participantes del Grupo E3. $f(\mathrm{C})=$ función contextual, $f(\mathrm{SU})=$ función suplementaria, $f(\mathrm{SE})=$ función selectora, $\mathrm{P}$ = participante. Las barras muestran la ejecución en preprueba y prueba - de izquierda a derecha, respectivamente-; los círculos y cuadrados representan la ejecución en las sesiones de entrenamiento; y la línea horizontal discontinua ubicada en el índice de ajuste de 0.8 indica el criterio de ajuste para pasar a la siguiente sesión. 
Finalmente, en las Figuras 8, 9 y 10 se muestran los resultados de los grupos controles, quienes no recibieron entrenamiento, pero sí se expusieron a prepruebas y pruebas. En general, las figuras muestran que todos los participantes obtuvieron un IA por debajo o cercano a cero en el primer $\mathrm{AC}$ al que fueron expuestos. Por otra parte, al enfrentar las pruebas de los siguientes AC, algunos participantes incrementaron el IA, aunque, a diferencia de lo registrado en los grupos experimentales, ninguno alcanzó el criterio de ajuste ni mostró incrementos progresivos y sostenidos al acumular experiencia en las siguientes pruebas.
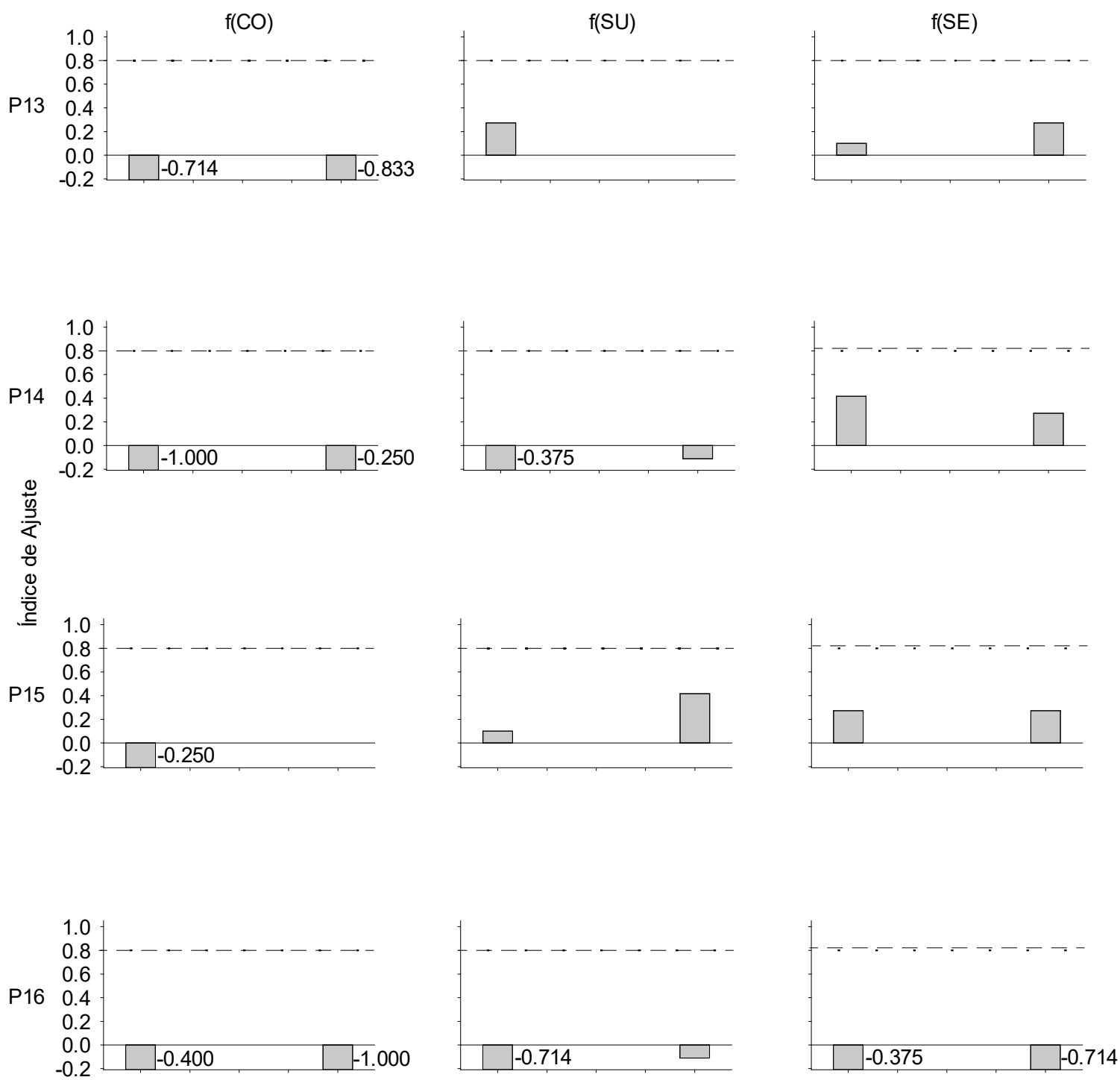

Figura 8. Índices de ajuste logrados por los participantes del Grupo C4. $f(\mathrm{C})=$ función contextual, $f(\mathrm{SU})=$ función suplementaria, $f(\mathrm{SE})=$ función selectora, $\mathrm{P}=$ participante. Las barras muestran la ejecución en preprueba y prueba de -izquierda a derecha, respectivamente-; los círculos y cuadrados representan la ejecución en las sesiones de entrenamiento; y la línea horizontal discontinua ubicada en el índice de ajuste de 0.8 indica el criterio de ajuste para pasar a la siguiente sesión. 

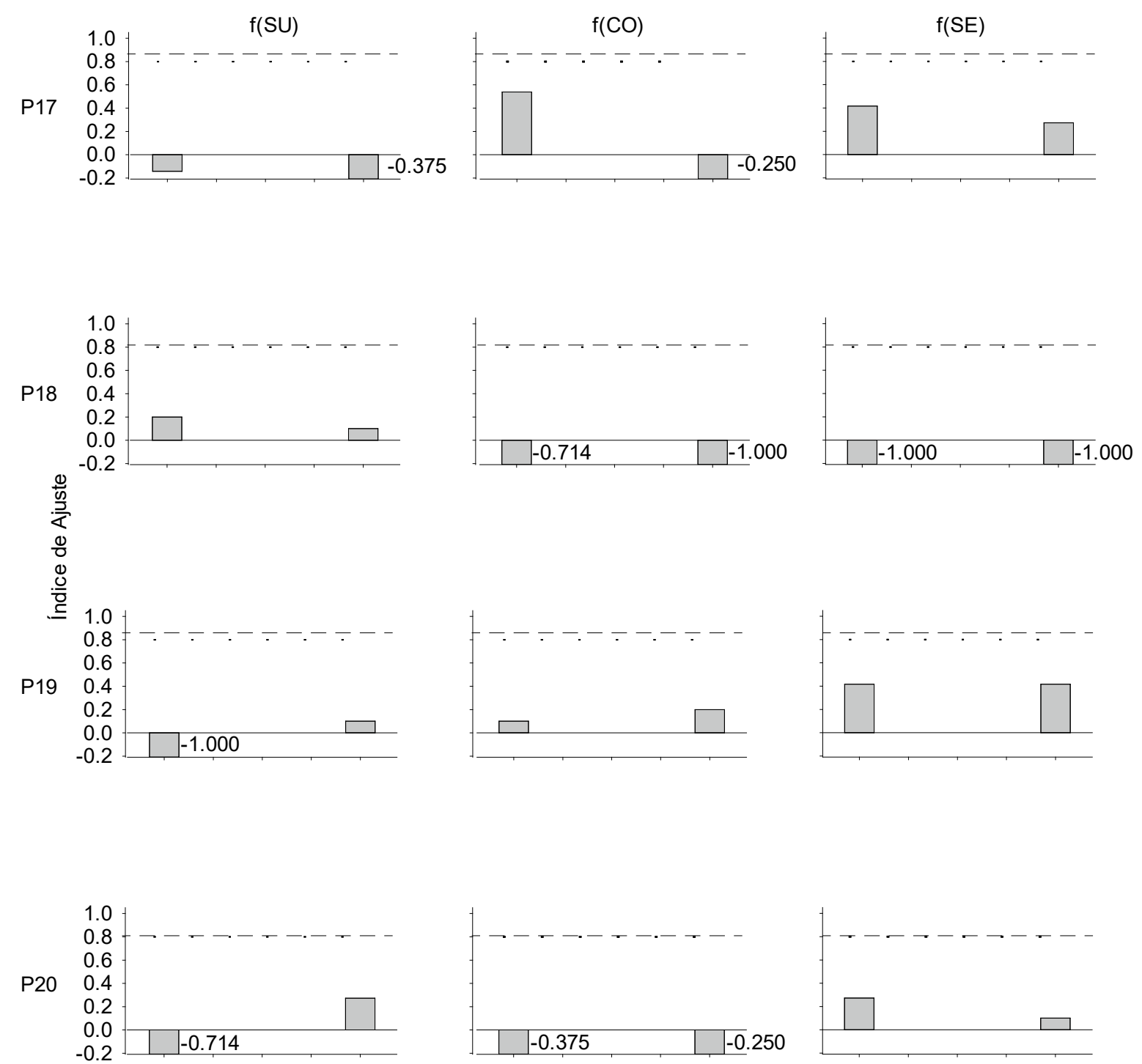

\section{Sesiones}

Figura 9. Índices de ajuste logrados por los participantes el Grupo C5. $f(\mathrm{C})=$ función contextual, $f(\mathrm{SU})=$ función suplementaria, $f(\mathrm{SE})=$ función selectora, $\mathrm{P}=$ participante. Las barras muestran la ejecución en preprueba y prueba — de izquierda a derecha, respectivamente-; los círculos y cuadrados representan la ejecución en las sesiones de entrenamiento; y la línea horizontal discontinua ubicada en el índice de ajuste de 0.8 indica el criterio de ajuste para pasar a la siguiente sesión. 

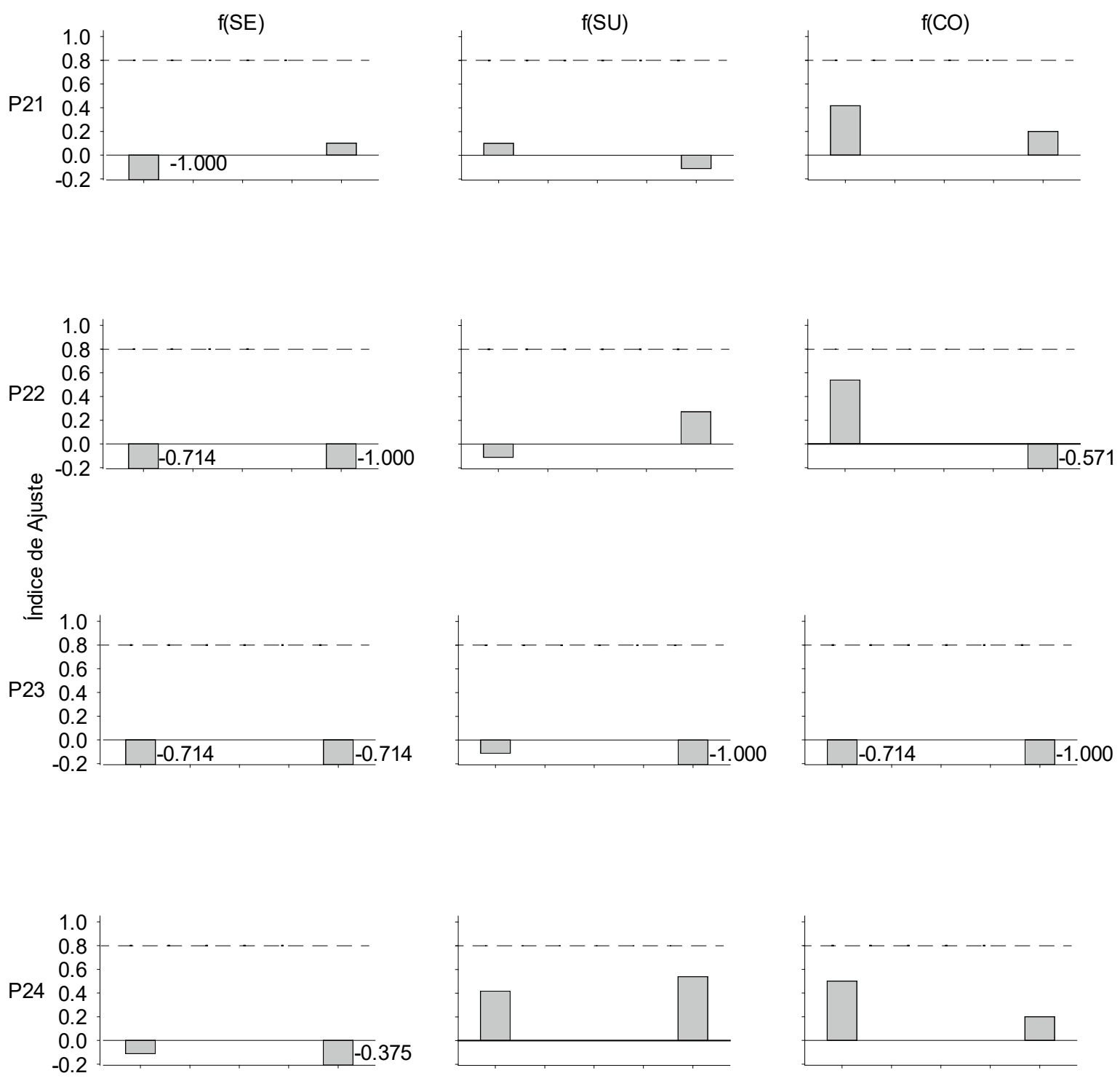

Sesiones

Figura 10. Índices de ajuste logrados por los participantes del Grupo C6. $f(\mathrm{C})=$ función contextual, $f(\mathrm{SU})=$ función suplementaria, $f(\mathrm{SE})=$ función selectora, $\mathrm{P}$ = participante. Las barras muestran la ejecución en preprueba y prueba — de izquierda a derecha, respectivamente-; los círculos y cuadrados representan la ejecución en las sesiones de

entrenamiento; y la línea horizontal discontinua ubicada en el índice de ajuste de 0.8 indica el criterio de ajuste para pasar a la siguiente sesión.

\section{Discusión}

Uno de los objetivos de la presente investigación fue evaluar el efecto de la historia de interacción sobre el ajuste conductual en tres niveles de organización funcional de distinta complejidad - contextual, suplementario y selector- . Al respecto, los resultados muestran que, a mayor nivel de complejidad en la organización funcional de la interacción, se requirió mayor cantidad de sesiones para que los participantes, en la fase en la que no tenían historia de interacción con las contingencias de la tarea, alcanzaran o superaran el índice de ajuste de 0.8. También, se encontró que la historia de interacción con las contingencias 
306

programadas en la tarea facilitó el aprendizaje del ajuste conductual en organizaciones funcionales de distinto nivel, independientemente de si la historia de interacción correspondía a uno o varios niveles de organización funcional de menor o mayor complejidad al entrenado y evaluado. En concordancia con esto, los grupos control, que no recibieron entrenamiento, no tuvieron un desempeño óptimo en ninguna de las pruebas de todos los niveles evaluados, esto teniendo en cuenta que el IA obtenido por los participantes fue cercano a cero.

Por otra parte, se encontró que, a mayor nivel de complejidad, mayor número de sesiones para mostrar aptitud funcional cuando los participantes carecían de historia de aptitud funcional previa al entrenamiento. En específico, del Grupo E1, que comenzó con el entrenamiento de diferenciación (contextual), solo un participante $(\mathrm{P} 2)$ requirió más de seis sesiones para lograr el criterio de aptitud funcional para pasar la prueba (superar el IA de 0.8); en el Grupo 2 , tres participantes requirieron más de seis sesiones para cumplir con el criterio de aptitud funcional de efectividad en la prueba (requiriendo de 9 a 12 sesiones); y, finalmente, el Grupo 3 fue el que mayor cantidad de sesiones requirió para lograr el criterio de aptitud funcional en la primera fase de entrenamiento que enfrentaron sus participantes, pues todos sus participantes requirieron más de seis sesiones para mostrar el nivel de aptitud funcional de precisión que se esperaba de ellos en la tarea (requiriendo de 10 a 15 sesiones) (véase Figuras 5, 6 y 7). Un resultado similar se ha encontrado en los estudios de Serrano (2008, 2009, 2016), en los que el incremento de la complejidad de la interacción se relacionó con un peor desempeño en la ejecución de los sujetos experimentales; sin embargo, esta similitud en los resultados debe analizarse en detalle, pues hay grandes diferencias entre este estudio y las investigaciones citadas respecto al método utilizado para medir y propiciar cada tipo de interacción.

Adicional a esto, se encontró que en el arreglo contingencial (AC) suplementario algunos participantes del Grupo E1 (P1) y E2 (P6, P7 y P8) requirieron dos o más sesiones de prueba para lograr el criterio de ajuste pese a que habían logrado dicho criterio en la última sesión de entrenamiento (véase Figuras 5 y 6). Quizás estos participantes aprendieron a establecer relaciones entre estímulos en tanto ocurrencias, sin diferenciar todas las asociaciones específicas símbolo-dibujo en las sesiones de entrenamiento, resultado que pudo deberse a las diferencias metodológicas entre el entrenamiento y las pruebas del AC suplementario, pues en el entrenamiento solo era necesario que el participante diferenciara que la respuesta a los símbolos para relacionarlos con algún dibujo, mientras que en la prueba era necesario diferenciar la relación específica entre cada símbolo y dibujo.

De este modo, pese a que en el AC suplementario los participantes relacionaban los dibujos con sus respectivos símbolos, al parecer las relaciones particulares que relacionaban dejaron de ser relevantes y solo "diferenciaron" que era necesario responder a los símbolos y no a las letras. De ser correcta la interpretación de estos resultados, cuando los participantes eran expuestos a la prueba del AC suplementario no diferenciaban las asociaciones particulares dibujo-símbolo, cuya aptitud era necesaria para responder de manera efectiva en la tarea. Al parecer, suplementar una relación entre estímulos no es suficiente para responder de manera efectiva, pues es necesario diferenciar la relación suplementada. No obstante, dado que la interpretación de estos resultados se hizo de manera indirecta, parece necesaria la elaboración de tareas experimentales que muestren datos que puedan relacionarse directamente con el supuesto de inclusividad funcional entre la función contextual y suplementaria.

Ahora bien, analizando en conjunto las secuencias ascendentes - las dos del Grupo E1 (contextual-suplementaria y suplementaria-selectora) y una del Grupo E2 (contextualselectora) - en cuanto a la historia interconductual se puede observar que el entrenamiento en un nivel de menor complejidad facilitó el desempeño en un nivel de mayor complejidad conductual. Al respecto, Velázquez y Flores (2013) encontraron datos relacionados con la facilitación del ajuste efectivo - mayor número de respuestas ante un tono asociado con la entrega de agua y menor número de respuestas por cada entrega de agua - en participantes con historia de aptitud funcional contextual - preexposición a la relación tono-agua-; sin embargo, los autores consideraron que en su investigación la historia de aptitud funcional contextual no fue una condición necesaria para el establecimiento de la función suplementaria, pues sujetos sin historia de aptitud funcional contextual también eran capaces de ajustarse de manera efectiva.

Hasta el momento, la evidencia encontrada, a la que se suma la de la presente investigación, sugiere que la historia de aptitud funcional de una función de menor complejidad parece facilitar el proceso de ajuste en funciones de mayor complejidad (Serrano, 2009, 2016; Velázquez \& Flores, 2013). No obstante, la evidencia es insuficiente para diferenciar dicha variable de otros factores que también podrían facilitar el ajuste - como el estado biológico del organismo o factores situacionales-. Así, también se considera pertinente mejorar los aspectos metodológicos que permitan dilucidar diferencias en la facilitación de la aptitud funcional en secuencias descendentes, pues los 
datos reportados no permiten diferenciarla de la facilitación encontrada en secuencias ascendentes.

Por otra parte, un estudio que contrasta con los resultados reportados y previamente citados es el de Ribes-Iñesta et al. (2009), en donde se encontró que la estructuración de funciones más complejas no requiere como condición necesaria la estructuración de funciones de menor complejidad. Sin embargo, también es posible identificar diferencias metodológicas entre los estudios que vale la pena revisar en detalle, como que los participantes del estudio tenían experiencia previa en la asociación de figuras geométricas, dada su escolaridad (estudiantes universitarios), que pudo facilitar su ajuste en las tareas; en ese sentido, se sugiere que las asociaciones entrenadas no formen parte del repertorio conductual de los participantes previo a la experimentación (González-Becerra \& Ortiz, 2014), tal como se hizo en esta investigación.

Un hallazgo parecido al de Ribes-Iñesta et al. (2009) se encuentra en el estudio de Tamayo y González-Becerra (2018), quienes realizaron un experimento con niños de educación primaria a quienes se expuso a secuencias de entrenamiento y pruebas en los niveles intrasituacionales. Después de entrenar el ajuste conductual en diferentes niveles de complejidad en asociación con las propiedades de velocidad y el número de alas de un "bicho", no se encontraron diferencias entre los grupos en cuanto al índice de ajuste, pues el ritmo de aprendizaje fue el mismo para el ajuste en todos los niveles de complejidad, independientemente de la historia interconductual de los participantes. Con respecto a este hallazgo, Tamayo y González-Becerra (2018) consideraron que la "aptitud numérica" de los participantes impidió el establecimiento de contingencias de función, lo cual pudo sesgar el contacto con el arreglo contingencial programado en cada tarea.

Ahora, si bien lo hasta aquí descrito abona al estudio de la complejidad e inclusividad progresiva propuesta en la taxonomía de Ribes y López (1985), con el fin de obtener mayor solidez y sistematicidad en los resultados e interpretaciones correspondientes, parece importante repensar y replantear algunos aspectos que estuvieron implicados en el presente estudio, entre ellos:

a. Desarrollar tareas experimentales que, además de evaluar y entrenar un tipo de comportamiento particular, permitan evidenciar la inclusión de funciones de menor complejidad - los trabajos de Serrano $(2009,2016)$ muestran avances al respecto-.

b. Identificar parámetros que puedan asociarse a la complejidad e inclusividad funcional, pues no se puede reducir el análisis al efecto de "facilitación" cuando hay otros elementos del sistema contingencial que podrían sumarse al estudio de las funciones psicológicas - como las secuencias de respuesta o el contacto parcial o total con los estímulos involucrados en la interacción-. Por ejemplo, se podría evaluar en los participantes que enfrentaron el entrenamiento de la función suplementaria si al responder a los símbolos, en vez de las letras, diferenciaban cada una de las relaciones dibujosímbolo a las que respondieron.

c. Precisar que la "manipulación" de la historia interconductual como "variable independiente" no implica considerarla como una condición necesaria para el ajuste, sino un factor que modula - facilita - dicho ajuste. Esta distinción permite evitar confusiones respecto al estatus que se le atribuye a dicha variable en la interacción, tal como ocurre cuando no se explícita su nivel de participación en el análisis del comportamiento (véase, p. ej., Okouchi, 2007; Pérez \& García, 2010).

d. Proponer medidas de análisis y métodos ad hoc a la teoría interconductual, tarea pendiente en la actualidad para los analistas de la conducta interesados en el análisis molar del comportamiento de corte interconductual, en el que algunos investigadores comienzan a explorar algunas propuestas (véase, p. ej., Camacho, 2017; Meraz \& Pérez-Almonacid, 2016; Serrano, 2009, 2016).

e. Y, por último, incrementar la evidencia empírica, realizando estudios con diseños de investigación que permitan evaluar distintos parámetros de los procesos de ajuste conductual en la estructuración de funciones psicológicas de distinto nivel de complejidad. Por ejemplo, una investigación subsecuente a la aquí reportada podría evaluar el efecto de la historia de la aptitud funcional en un nivel de complejidad sobre el índice de ajuste alcanzado en una función de complejidad distinta (ascendente o descendente), pero sin pasar por una fase de entrenamiento.

En conclusión, la reflexión teórica y la evaluación empírica de la correspondencia entre lo que se postula y lo que ocurre en la naturaleza de lo psicológico son actividades complementarias en el quehacer científico, de modo que una teoría sin evaluación empírica corre el riesgo de convertirse en un dogma, así como una investigación sin marco teórico limita el análisis a las particularidades que pueda develar el uso del sentido común. En este sentido, en el estudio de las funciones psicológicas propuestas por Ribes y López (1985) se dispone por el momento de algunos datos relacionados a su complejidad y relación inclusiva, aunque estos no permiten llegar a conclusiones contundentes. No obstante, a partir de los resultados de la presente 
investigación se puede contar con métodos, medidas, datos $\mathrm{y}$ análisis que sirven de base para futuras investigaciones.

\section{Referencias}

Camacho, I. (2017). Teoría de la conducta: Una discusión sobre las categorías de medida y metodología. Acta Comportamentalia, 25(3), 411-422. http://www.redalyc. org/articulo.oa?id=274552568008

González-Becerra, V., \& Ortiz, G. (2014). Efectos de la retroalimentación y el tipo de descripción de contingencias sobre la conducta de discriminación condicional en una tarea de igualación de la muestra. Revista Mexicana de Análisis de la Conducta, 40(1), 51-80. http://dx.doi.org/10.5514/rmac. v40.i1.63600

Kantor, J. R. (1924). Principles of psychology (Vol. 1). Principia Press.

Kantor, J. R. (1926). Principles of psychology (Vol. 2). Principia Press.

Kantor, J. R. (1959/1980). Psicología interconductual: Un ejemplo de construcción científica sistemática. Trillas.

Meraz, E., \& Pérez-Almonacid, R. (2016). Efecto de diferentes tipos de interacciones verbales sobre el ajuste lector. Revista Mexicana de Análisis de la Conducta, 42(3), 222248. http://dx.doi.org/10.5514/rmac.v42.i3.58839

Okouchi, H. (2007). An exploration of remote history effects in humans. Psychological Record, 57, 241-263. https://doi. org/10.1007/BF03395575

Pérez, T., \& García, M. (2010). ¿Cuál es el estatus del concepto "historia de reforzamiento", de uso frecuente en el análisis de la conducta? Revista Mexicana de Análisis de la Conducta, 36(3), 77-91. http://dx.doi.org/10.5514/rmac. v36.i3.21398

Ribes-Iñesta, E. (2001). Funciontal dimensions of social behavior: theoretical considerations and some preliminary data. Mexican Journal of Behavior Analysis, 26(2), 285-306. http://rmac-mx.org/functional-dimensions-of-social-beha vior-theoretical-considerations-and-some-preliminary-data/

Ribes-Iñesta, E. (2004). Acerca de las funciones psicológicas: un post-scriptum. Acta Comportamentalia, 12(2), 117127. http://www.revistas.unam.mx/index.php/acom/article/ view/14599

Ribes-Iñesta, E. (2007). Estados límites de campo, medios de contacto y análisis molar del comportamiento: reflexiones teóricas. Acta Comportamentalia, 15(2), 229-259. http://www.revistas.unam.mx/index.php/acom/article/ view/14522

Ribes, E., \& López, F. (1985). Teoría de la conducta: un análisis de campo y paramétrico. Trillas.

Ribes-Iñesta, E., Rangel, N., \& López-Valadéz, F. (2008). Análisis teórico de las dimensiones funcionales del comportamiento social. Revista Mexicana de Psicología, 25(1), 4557. http://www.redalyc.org/articulo.oa?id=243016300003

Ribes-Iñesta, E., Vargas, I., Luna, D., \& Martínez, C. (2009). Adquisición y transferencia de una discriminación condicional en una secuencia de cinco criterios de ajuste funcional. Acta Comportamentalia, 17(3), 299-331. http://www. revistas.unam.mx/index.php/acom/article/view/18156

Serrano, M. (2008). Análisis experimental de las funciones contextual, suplementaria y selectora: efectos de la concurrencia intra e intercontingencial (Tesis doctoral no publicada). Universidad de Guadalajara, México.

Serrano, M. (2009). Complejidad e inclusividad progresivas: algunas implicaciones y evidencias empíricas en el caso de las funciones contextual, suplementaria y selectora. Revista Mexicana de Análisis de la Conducta, 35(2), 161-178. http://dx.doi.org/10.5514/rmac.v35.i0.16202

Serrano, M. (2016). Ajuste comportamental bajo diferentes condiciones de entrega de agua Acta Comportamentalia, 24(1), 5-13. http://www.revistas.unam.mx/index.php/acom/ article/view/54708

Schoenfeld, W. N., \& Cole, B. (1972). Stimulus schedules: The t-tau system. Harper and Row.

Tamayo, J., \& González-Becerra, V. (2018). Evaluación de la facilitación interfuncional en las funciones intrasituacionales. IPyE: Psicología y Educación, 12(24), 18-35.

Torres, C., Ortiz, G., Rangel, N., \& González, V. (2012). Análilsis del concepto situación en la estructuración de las funciones psicológicas. En M. Padilla \& R PérezAlmonacid, La función sustitutiva referencial: análisis histórico-crítico/avances y perspectivas, (pp. 149-168). University Press of the South.

Velázquez, G., \& Flores, C. (2013). Contribución de contingencias entre estímulos sobre la efectividad del comportamiento en la estructuración de campos suplementarios. Acta Comportamentalia, 21(4), 425-434. http://www.revistas. unam.mx/index.php/acom/article/view/43607 